L'HOMME L'Homme

Revue française d'anthropologie

$230 \mid 2019$

Varia

\title{
Enquêter auprès des morts
}

\section{Vinciane Despret}

\section{(2) OpenEdition}

Journals

Édition électronique

URL : https://journals.openedition.org/lhomme/33844

DOl : 10.4000//homme.33844

ISSN : 1953-8103

\section{Éditeur}

Éditions de l'EHESS

\section{Édition imprimée}

Date de publication : 13 juin 2019

Pagination : 5-26

ISSN : 0439-4216

\section{Référence électronique}

Vinciane Despret, «Enquêter auprès des morts », L'Homme [En ligne], 230 | 2019, mis en ligne le 01

janvier 2022, consulté le 08 janvier 2022. URL : http://journals.openedition.org//homme/33844 ; DOI : https://doi.org/10.4000//homme.33844

(c) École des hautes études en sciences sociales 



\title{
Enquêter auprès des morts
}

\author{
Vinciane Despret
}

66

LES MORTs sont des gens comme les autres». Cette proposition m'a été offerte par Philippe, qui est, depuis quelques années, médium dans le cercle spirite de la ville où j'habite. J'ai assisté à de nombreuses séances de ce groupe, le plus souvent sous la conduite de sa mère, Michèle Willemsem, qui est médium également. Cette réflexion, "Les morts sont des gens comme les autres", m'a été livrée il y a quelques mois, lors d'un entretien auquel je les avais invités tous les deux, chez moi. Au cours de cette longue conversation, je leur avais demandé de m'expliquer la manière dont ils entraient en communication avec les défunts : qu'est-ce qu'entendre, sentir ou voir signifient, dans ce cas? Nous avons évoqué leur histoire, surtout celle de Michèle, étonnamment comparable à celles que les ethnologues qui se sont intéressés à ces destins peu communs ont décrites, avec ces épreuves, ces mises en danger du corps et ces démêlés avec la mort, ces sentiments de présence et ces surprenants rêves prémonitoires. Des destins que ces personnes ont réussi à socialiser et ont su transformer en destinée.

"Les morts sont des gens comme les autres», me disait donc Philippe. En relisant cette phrase, je me suis souvenue que j'avais déjà rencontré des formulations très semblables, au cours de mes recherches. Ainsi avais-je pu lire l'éditorial du New York Times du 24 avril 2014, intitulé «Neanderthals are People, Too ». Son auteur, le généticien suédois Svante Pääbo, directeur du Département d'anthropologie évolutionnaire du Max Planck Institut à Leipzig et responsable du projet international de séquençage du génome 
de Néanderthal, s'opposait fermement au projet de son collègue, George Church, de cloner un Néanderthalien. Ce qui est plus étonnant, c'est que l'article commence par l'évocation du grand-père de l'auteur, mort de la grippe espagnole en 1919, à l'âge de 30 ans. Ses enfants et petits-enfants n'ont pas pu le connaître, poursuit Pääbo. "Il était un brillant mathématicien », explique-t-il, et, "scientifique moi-même, je serais curieux de savoir comment il était». "Mais», ajoute-t-il encore, «je me console en pensant qu'il continue à vivre en moi» ${ }^{1}$.

Vous pourrez remarquer avec moi que, quand j'essaie de m'éloigner de mon sujet, je me fais aussitôt rattraper. C'est souvent le cas avec les morts. Précisons encore, et cela ne nous en éloignera pas davantage, que l'enrôlement du grand-père dans l'argument n'est pas gratuit: "Souhaiterais-je», écrit Pääbo en conclusion de son article, «exhumer les os de mon grandpère pour recréer, à partir d'eux, un jumeau monozygote qui vivrait ainsi quatre-vingt-quinze ans après la mort de son aîné? La réponse est clairement non. Et comme ma réponse est non, pourquoi en irait-il autrement pour Néanderthal?». Les morts sont des gens aussi - en tout cas s'il s'agit de morts humains.

Mais les non-humains peuvent aussi faire l'objet de cette requalification, et devenir des gens. Et c'est à nouveau dans le New York Times que j'en avais, au hasard de mes recherches sur les animaux, trouvé une illustration, plus précisément dans un article du 5 octobre 2013, intitulé: «Dogs Are People, Too ». Il était cette fois signé par un spécialiste en neurologie, Gregory Berns. Berns décrit le travail de son équipe avec des chiens. Ils cherchent à savoir ce que les chiens pensent, et peut-être même, précise-t-il, ce qu'ils pensent de nous, les humains. Comme les chiens ne parlent pas, les scientifiques ont décidé d'explorer la manière dont leur cerveau fonctionne au moyen de la technique de l'imagerie cérébrale, et ce, afin d'établir les similitudes possibles. Et ils ont découvert, que ce fonctionnement était très similaire à celui des humains. Est-ce cela qui fera des chiens des gens comme nous? En réalité, je ne crois pas que ce sont ces ressemblances cérébrales qui déterminent la possibilité de ce rapprochement, mais bien plutôt la manière dont elles ont été construites. Quand des similitudes sont construites en laboratoire, j'ai appris qu'il fallait s'intéresser au dispositif qui a permis de les faire émerger. Et de fait, le dispositif est assez étonnant: Gregory Berns et son équipe ont organisé des formes de stage, pour chiens volontaires, au cours desquels ces chiens apprennent à rester dans le PET scan (à croire que la machine a été créée pour eux). Quand je dis "chiens volontaires", je fais référence à plusieurs caractéristiques de la procédure expérimentale:

1. Tous les extraits d'articles de presse ou d'ouvrages en anglais ont été traduits par mes soins. 
les scientifiques ont créé un formulaire de consentement éclairé, semblable à celui que les parents doivent signer lorsque leurs enfants participent à des recherches. Dans ce formulaire, est indiqué le fait que la participation doit être volontaire, et que le chien a le droit de quitter l'expérience dès que le maître aura jugé qu'il le désire. Et si le chien lui-même exprime cette volonté, s'il ne veut pas aller dans la machine ou s'il manifeste l'envie d'en sortir, il sera exempté d'expérience. Les ethnologues Aude Michelet et Charles Stépanoff, dans un très bel article, ont avancé que l'anthropomorphisme nous a rendus humains et que notre capacité à imputer des motivations, des perspectives et des émotions à d'autres espèces aurait sans doute joué un rôle dans ce qu'on appelle le "succès évolutif» de notre espèce. Mais, observentils, l'anthropomorphisme a également des effets sur l'anthropomorphisé: du fait d'être traités par les humains comme des alter ego, "les chiens ont fini par leur ressembler» (Michelet \& Stépanoff 2016: 45). À noter aussi que l'anthropomorphisme participe pleinement encore du devenir "personne» au sein même de notre espèce, puisque c'est en traitant les petits d'humains en humains que ces derniers deviennent des personnes comme les autres, c'est-à-dire différents comme les autres. Ce sont des pratiques d'engendrement, si je suis Luc Boltanski, ou d'instauration, si je me réfère à Étienne Souriau.

Je remarquerai en passant que ces trois cas de figure, ces morts familiarisés, ces chiens anthropomorphisés et ce Néanderthal «réhumanisé», présentent comme caractéristique commune, mais aussi comme différence, l'usage de technologies particulières. Je viens de le développer pour les chiens, en montrant les effets du dispositif lui-même dans la construction de la ressemblance; ce déploiement technologique est évident aujourd'hui pour Néanderthal, surtout depuis les tout récents travaux des archéologues et des paléontologues qui, grâce aux techniques de fouille et de décryptage, mais aussi grâce aux techniques de séquençage du génome mises en œuvre par l'équipe de Pääbo, ont pu remettre en cause l'image de brute épaisse que portait Néanderthal, et dont je suspecte, avec d'autres, qu'elle permettait à l'exceptionnalisme humain d'assurer son inquestionabilité. Les techniques des médiums, quant à elles, s'avèrent également constituer des technologies de réduction de la distance, ne serait-ce que parce que, concrètement, elles ambitionnent de rendre présent. Ce sont des dispositifs de convocation. Mais ce n'est pas tout. Et c'est sous le signe de ce «ce n'est pas tout» qui scandait merveilleusement le travail de Lévi-Strauss, que je voudrais inscrire ce qui va suivre.

Mais ce n'est pas tout. Car s'il s'agit bien, en prenant acte (ou en mettant en acte) que les morts sont des gens comme les autres, d'opérer la réduction de la distance ou de construire des formes de proximité. Mais la proposition 
de Philippe signale autre chose. Si elle insiste, avec ce "comme les autres", sur la familiarité des rapports avec les morts, c'est-à-dire sur le fait que l'on puisse avoir des relations avec les morts qui présentent le même caractère de familiarité que l'on peut avoir avec les vivants, elle indique en même temps que les morts sont, comme le sont les vivants, différents les uns des autres. En témoigne, par exemple, le fait que Michèle rappelle, lors des séances où les personnes viennent pour demander une mise en rapport avec un défunt: "[qu'] on ne sait jamais sur qui on peut tomber", reprenant les mêmes mots que ceux que l'ethnologue Christophe Pons (2002) a entendus lors de son enquête en Islande. Cette différentiation s'avère d'ailleurs réciproque: nous sommes également des personnes pour les morts, non seulement parce que ceux dont les médiums assurent la présence s'adressent à des personnes particulières et bien identifiées (fils, filles, petits-enfants, nièces, neveux ou amis), mais aussi parce que, et de manière remarquable, les morts se montrent attentifs à la personnalité même du médium. Ainsi, lorsque nous évoquions les registres sensoriels par lesquels les médiums recevaient les messages, Philippe me confia, non sans humour:

"J'ai pu constater que les esprits ont fait des études de pédagogie. On sait que pour tenir une classe, il faut mobiliser tous les registres sensoriels qui sont distribués chez les élèves (il y a les visuels, les auditifs, les kinesthésiques...) et donc l'instituteur doit bouger, parler, écrire... Dans mon cas, j'ai une tendance auditive, et c'est ainsi que les messages généralement m’arrivent, les messages visuels étant très pauvres chez moi. Les esprits font des efforts pour fournir le message dans le registre sensitif du médium auquel ils s'adressent».

On observera que la manière dont Philippe a formulé son constat présente une caractéristique familière à tous ceux qui ont travaillé avec les médiums. Il y a en effet une redistribution de la puissance d'agir avec, comme corollaire, un effacement délibéré de la personne du médium, sa mise en retrait: il doit pouvoir être habité par un autre être et doit, pour ce faire, laisser la volonté de ce dernier se substituer à la sienne. Cet effacement est la condition de réussite du dispositif. Il peut prendre plusieurs formes, qu'ont repérées aussi bien Maurice Bloch (1993) que Giordana Charuty (2002) ou Christine Bergé (1990). Mais toutes ces formes manifestent que le médium est étranger à ses productions, il n'est donc qu'un canal, ce qui ratifie la présence tierce. En témoigne par exemple le fait que, parfois, le médium ne comprend pas le message qu'il est en train de transmettre. J'ajouterai en passant que ce mode opératoire met toujours celui qui consulte au travail de l'interprétation, donc de la pensée, ce qui, à mon sens, rattache ce dispositif aux procédés thérapeutiques. J’y reviendrai ultérieurement. 
Toujours est-il que la non-compréhension du message par celui qui le transmet atteste d'une mise en rapport particulièrement réussie avec le défunt. Et elle le sera d'autant plus, les médiums le disent clairement, si ce message trouve une prise dans le réel. Car c'est bien ce que va faire le message: chercher une prise. Et mettre donc celui à qui il est destiné en quête de cette prise possible, ce qui veut dire au travail de l'enquête: que veut-il me dire? Qu'attend-il de moi? Une enquête avec un mort. En d'autres termes, le message, en attente de signification, en quête de prise, le message comme énigme, met son destinataire en mouvement et au travail de la pensée. Il va produire un certain type d'attention et un autre régime d'affect - surtout si le message ne prend pas immédiatement sa signification. J'y reviens déjà. C'est là, me semble-t-il, une dimension thérapeutique essentielle du dispositif: non sa condition possible de réussite, mais sa possible réussite dans ses effets. Mais cette version de l'histoire est la mienne, pour le médium, la question de la réussite tient d'abord, en amont, en sa possibilité d'avoir constitué un relais fiable. "Le message», me dit Philippe, «n'est jamais aussi probant que lorsque nous sommes autant surpris que les autres: le message prend, à certaines occasions, des voies tellement détournées qu'il ne recevra sa signification que lorsqu'il se réalisera dans le réel, c'est le réel alors qui lui donne son sens, rétroactivement». Philippe raconte qu'il était en train de transmettre un message avec, poursuit-il - ce qui est plus que fréquent - beaucoup de banalités: «Repose-toi», "Occupetoi de toi». Toutefois, à la fin du message, il demande aux personnes s’il y a un problème avec la voiture. Devant leur réponse négative, il leur dit: "Je dois transmettre. Je ne veux pas vous inquiéter, mais s'il y a quelque chose, pensez-y avant de démarrer". Une ou deux semaines plus tard, le couple revient pour le remercier: il a sauvé la vie de l'épouse. À la suite du message, ils ont fait réviser leur voiture et l'épouse a pris la camionnette de chantier pour ses déplacements. Or, son véhicule a été embouti assez violemment, sans conséquence, vu la hauteur du siège du conducteur. Dans une voiture, en revanche, cet accident aurait eu des suites autrement plus graves, et elle aurait été pour le moins grièvement blessée. "Ce message a permis ", dit Philippe, "par une série de coïncidences, si on pense que ce sont des coïncidences, et au départ d'un message banal, que les choses soient changées».

En laissant la question des coïncidences ouvertes, et en démontant un enchaînement somme toute très aléatoire et susceptible de bien d'autres reconstructions, Philippe traduit en réalité une dimension de ces situations sur laquelle, je crois, on n'a pas assez insisté: le fait qu'elles sont non seulement toujours marquées par une forte indétermination, mais surtout une indétermination dont le médium est très conscient. Et je me rends compte, 
en le relisant, que le dispositif médiumnique met en œuvre une ontologie, et une épistémologie du rapport de la vérité au réel, d'une sophistication remarquable: c'est le réel qui donne son sens, c'est-à-dire c'est le réel qui donne sa vérité, à l'annonce. En d'autres termes, c'est dans le réel que l'annonce cherche, et parfois trouve, les conditions de sa réalisation. Selon William James, la vérité est ce qui advient à l'énoncé; un énoncé en quête de vérité participe à un réel en train de se faire. Et il met activement à contribution, dans ce faire de la réalité en train de se faire, le porteur de l'énoncé en quête de sa vérité (1913 [1909] : 69; 1948 [1911] : 263). On n'est pas, loin de là, dans le régime de la preuve, mais, plutôt, dans celui de l'épreuve. Car il s'agit bien d'une épreuve: une annonce lance son destinataire dans l'enquête de sa réalisation. C'est le propre des énigmes. À l'énigme, une seule réponse possible, pour qui sait l'accueillir: qu'est-ce qu'on fait avec cela? C'est la question que j'ai le plus souvent entendue dans les histoires que m'ont racontées les personnes confrontées à la question d'un défunt. Et cela me semble indiquer qu'elles avaient saisi que ce qui les attendait était de l'ordre de l'énigme.

L'énigme de l'annonce, telle que la conçoit Philippe, dès lors, remplit admirablement les conditions de félicité que William James assignait à la vérité: dans sa quête de devenir vrai, de sa réalisation, un énoncé apporte sa contribution au réel, sur le mode d'une augmentation. Et, plus remarquable encore, cette réalisation n'équivaut pas à une résolution, puisque l'indétermination qu'elle portait se retrouve, quoique transformée, à l'aboutissement de sa réalisation. En témoigne la perplexité qui accompagne celle-ci : doit-on appeler cela une série de coïncidences? L'accident aurait-il eu lieu si on avait gardé la voiture? La réalité prête à l'énoncé sa signification, et je dis bien "prête", car celle-ci pourra toujours être remise au travail de la pensée, mais elle n'épuise ni les multiples sens de cet énoncé ni le sens de leurs rapports. "Le propre d'une indétermination activement cultivée», écrivait Isabelle Stengers, «est de susciter d'autres indéterminations d'entrer en résonance avec d'autres modes de mise en culture» (2007: 44). C'est la marque de toutes les histoires que l'on m'avait racontées, au temps où je menais mon enquête, et de celles qui me sont parvenues après la fin de celle-ci, en réponse à cette dernière.

Le jeune ethnologue Paul Sorrentino a enquêté, au cours de ces dernières années, sur l'invention d'un nouveau rituel de possession au Vietnam. Des personnes apprennent, dans le cadre d'un rituel, le rituel àp vong, à accueillir et à se laisser posséder par l'âme de l'un de leurs disparus, permettant ainsi à ce dernier d'exprimer des volontés qui n'auraient pas été accomplies, de prendre soin de la famille, de donner de ses nouvelles, voire, ce qui est précieux dans un pays longtemps en guerre où quantité de morts n'ont 
pu être retrouvés, de les guider dans la recherche de leurs corps. Dans les dernières lignes des remerciements qui ouvrent son livre, Sorrentino écrit que certains des passages ont été rédigés dans la chambre de l'hôpital où, je le cite, «[son] père se préparait à un combat qu'il n'eut finalement pas le temps de mener» (2018: 11).

«Lors de notre dernière conversation, je lui avais annoncé le dépôt de mon manuscrit et le début du travail éditorial dont ce livre est l'aboutissement. Pendant que je l'écrivais, j'ai appris qu'on pouvait rendre un mort présent en s'adressant simplement à lui. Alors voilà: c'est fini» (Ibid.).

«Alors voilà: c'est fini.» Ces mots, au-delà du fait qu'ils m’ont touchée bien plus que je ne pourrai le dire dans ce cadre, m’ont renvoyée aux toutes dernières lignes de l'épilogue de ce même livre, lorsque, devant la multiplicité des versions possibles et contradictoires d'une même histoire, celle de la possession de Kien par le grand-père de son épouse, Sorrentino conclut: "Les enquêtes ethnographiques, comme les épreuves de la possession, ne sont jamais vraiment closes" (Ibid.: 320).

Ce n'est pas fini, parce que ce n'est jamais fini. Car tel est bien le parti pris de Paul Sorrentino, confronté à la question de la vérité de la possession par un défunt - est-on vraiment possédé? est-ce vraiment le mort? -, le parti pris d'apprendre, avec ceux qui en font l'expérience, à ne pas fermer cette question sur une réponse univoque. Plutôt chercher une prise, celle qui s'accordera au mieux avec ces expériences qui rendent perplexes tous ceux qui s'y engagent, une prise qui fera honneur à cette perplexité et à l'intelligence qu'elle met en ouvre, une prise à la hauteur d'une expérience qui s'inscrit d'autant moins dans les catégories étroites et binaires de la croyance qu'elle ne cesse de déborder celles de la critique. Cette prise, Sorrentino choisira avec sagesse de l'exercer à partir des conditions écologiques qui rendent ces expériences possibles, les milieux dont elles peuvent se nourrir pour exister. Car la question de la vérité peut constituer un véritable poison pour ces situations, surtout, Isabelle Stengers nous l'a appris, lorsqu'elle se pose en ces termes: «Est-ce vraiment?» (2014: 154). Et pourtant, elle ne cesse de se poser, souvent sur le mode, qui est notre bêtise à nous, du «ou bien... ou bien": ou bien tu te dis "vraiment possédé», ou bien tout cela n'est qu'un produit de ta subjectivité. Ce qu'il faut donc apprendre, et c'est là la belle leçon d'écologie des pratiques qu'elle nous a enseignée, c'est à chercher le régime pertinent dans lequel un énoncé ou un acte ou une expérience rencontre le réel, et devient vrai. Garder d'autant plus précieusement la question de la vérité, et je cite à nouveau Paul Sorrentino, que ceux qui s'engagent dans cette expérience de la possession l'«abordent tant avec discernement et sens critique qu'avec les émotions et les attentes que leurs trajectoires 
biographiques ont construites en amont de leur engagement dans un rituel de àp vong» (2018: 33).

Et c'est donc à cultiver l'hésitation, l'indétermination si remarquables de ces situations que l'ethnologue est convié. Et de ce fait, Sorrentino, au cours de son enquête, va apprendre à transformer l'impossibilité de jamais connaittre «le fin mot de l'histoire» (Ibid.: 145), à transformer cette impossibilité en ce que j'appellerais une "éthique des récits». Amor fati.

$\mathrm{Ne}$ pas chercher le fin mot de l'histoire. Je viens d'évoquer, à propos de cette enquête au Vietnam, une possible "éthique des récits». Ce terme que son travail m'inspire, je l'appliquerai aujourd'hui à cette histoire si intéressante qui me semble avoir guidé la lecture que l'anthropologue Heonik Kwon propose des fantômes dans le Vietnam contemporain. Dans la région de Da Nang, raconte-t-il, l'hôte de passage reçoit généralement, à la fin de la conversation, un verre d'eau du puits dont on lui dit qu'il est offert par un esprit. Ce premier verre d'eau est légèrement salé. Les suivants, puisés au même réservoir, ne le seront pas. Tout le monde ne peut goûter le sel de cette eau. Or, Kwon s'inquiète: il avoue n'avoir connu l'expérience du sel que de rares fois (2008: 104). Comment une eau de puits peut-elle avoir la saveur de l'eau de mer? Pourquoi pour certains et pas pour d'autres? Est-ce mon âme ou mon corps, se demande Kwon, qui reconnaît le sel? Et si quelqu'un le reconnaît et d'autres pas, quel est celui qui a un problème, lui ou eux? Ces questions ne demandent pas de solution, quoiqu'on puisse toujours y répondre. C'est là leur force performatrice. Kwon en prend acte lorsqu'il avoue que, à la question de savoir si le sel est dans l'eau ou dans la bouche (pour éviter de dire, dans la tête), s'il est normal ou non de la goûter, anormal de le faire ou de ne pas le faire, et comment comprendre cet étrange phénomène, il n'a, aucune réponse raisonnable. «Mais», continue-t-il, «à la place des réponses, l'expérience du sel spirituel m’a conduit à d'autres événements et à d'autres histoires, et ma soif de devenir capable de goûter comme les autres a ouvert de nouveaux accès pour comprendre ces événements et ces histoires [...]. Un proverbe fréquemment évoqué au Vietnam est: "Les ancêtres ont mangé trop de sel, leurs descendants désirent de l'eau" " (Ibid.: 105).

Ce proverbe, qui peut être utilisé dans des contextes très différents, peut avoir quantité de significations. L'une d'elles se rattache à cette histoire:

«[...] les vrais désirs humains, dit ce proverbe, ne sont pas ceux d'un individu isolé. L'origine du désir est, comme le sel de l'eau offerte par l'esprit, chez quelqu'un d'autre, et ce n'est qu'en présence de cet autre que l'eau devient salée. Le désir de se souvenir et de commémorer, de même, peut être un désir qui émerge entre le passé et le présent, et qui est partagé entre celui qui se souvient et celui dont on se souvient" (Ibid.: 105-106). 
Sont-ce les morts qui réclament le besoin d'être souvenus? Est-ce nous qui leur imputons ce désir? Ne pas donner le mot de la fin, c'est respecter ce que Gilles Deleuze appelait un "agencement». C'est un rapport de forces qui fait tenir un événement; le désir d'être souvenu et le désir de se souvenir «tiennent» ensemble, il n’y a pas de précédence. Démembrer cet agencement, accorder une priorité ontologique à l'imaginaire des vivants - «ce serait lui la cause réelle de ce désir»-, ou à la volonté des morts, lui ôterait toute signification, détruirait ce qu'Étienne Souriau désignait comme son "éclat de réalité", sa force ontologique propre, sa "manière d'être » en tant qu'agencement (1939: 10).

$\mathrm{Si}$ je revisite aujourd'hui cette histoire qui témoigne d'une éthique des récits, c'est parce qu'elle me semble traduire non seulement un consentement à ne pas imposer le fin mot de l'histoire, mais qu'elle atteste également de la volonté de transformer activement ce consentement en une obligation: celle de donner suite. Une obligation à imaginer, à penser, à honorer le refus de l'histoire de se clôturer.

"Ce n'est pas tout.» Ou, si je reprends à Eduardo Viveiros de Castro la belle formule qu'il offre en retour à Claude Lévi-Strauss: «Ce n'est pas tout, donc, car rien n'est jamais tout» (2008: 130). Ce n'est pas tout, parce que Heonik Kwon accepte l'épreuve de l'énigme, qui n'attend pas une résolution, mais, au contraire, une suite. Reformuler la question pour celle-ci : qu'est-ce que je fais avec cela? Comprendre ne devrait jamais vouloir dire autre chose - comprendre comme "prendre avec», comme on dit "faire avec». C'est cela, donner une suite, et je reprends à présent ce que Mathieu PotteBonneville, s'inspirant de Wittgenstein, assignait au terme "comprendre ${ }^{2}$ : si on te demande le rapport entre deux, quatre, huit, seize, tu manifestes que tu as compris en proposant trente-deux. Tu donnes une suite. Car ce qui caractérise les récits que font faire les morts, c'est que, justement, ce n'est jamais fini. Au contraire, ces récits sont une protestation contre ce qui se donne comme fini. C'est ce qui me semble ressortir de toutes les histoires que j'ai pu recueillir, que ce soit dans leur forme, ou dans la suite qu'elles suscitent. Il y a redistribution constante des perspectives et des puissances d'agir. Ce sont des technologies de re-convocation. Les récits, en d'autres termes, re-suscitent.

À l'appui de cette hypothèse, je me suis rendu compte que les récits que j'avais relayés, que ce soit dans des publications ou des conférences que j'ai pu faire au cours de mon enquête, avaient le même effet. Début 2015, mon enquête était en principe terminée, du moins tel était mon souhait; j'allais

2. Cf. l'émission radiophonique «La grande table» sur le thème «Faut-il faire son deuil ? », diffusée sur France Culture, le 2 décembre 2015. 
me tourner vers d'autres vivants accompagnés d'autres êtres, pourquoi pas les oiseaux? C'est bien intéressant aussi les oiseaux, et ce sont d'autres modes également bien prometteurs de faire communiquer la terre avec les cieux. Mais les morts ne l'entendent pas de cette oreille. Non seulement les morts faisaient faire des choses aux vivants, cela avait été le motif de ma recherche, mais à présent, les morts des uns faisaient faire des choses aux vivants des autres. Ainsi, rien que dans la deuxième semaine de septembre, alors que je préparais cette conférence et que je me remettais à de nouvelles lectures découvertes après-coup, je reçus deux courriels. L’un de Bernard Féry qui me disait qu'à la lecture des histoires partagées, il se rendait compte que le roman qu'il avait écrit, La Veuve du procureur (2018), suivait l'intuition de nombre de personnes qui se sentent tenues d'accomplir ce que le mort ne peut plus faire. L'autre, de Christophe Laurens, m'annonçait qu'il avait perdu son père cet été. Il continuait: «et j'ai pensé à toi en lui choisissant des bonnes chaussures pour se promener dans l'au-delà». Christophe me demandait également si j'avais vu le documentaire Carré $35^{3}$ et me rappelait qu'on y voit à un moment une inscription funéraire magnifique qui confie aux oiseaux le soin de transmettre les pensées des vivants à une petite fille morte trop tôt. Je ne me souvenais pas de cette inscription, mais elle m'a rappelé un courriel reçu fin 2015 de Jean-Christophe Janin qui me racontait que des personnes avec qui il entretenait une correspondance avaient placé, sur la tombe de leur jeune fille décédée, une boîte aux lettres. Depuis, ils reçoivent régulièrement des messages de personnes qui aiment Juliette, et même d'inconnus. Ainsi avait-il conlut, «Elle vit toujours!». Il avait ajouté que sa maman avait alors lancé: «C'est dur d'élever un enfant mort!», et avait commenté: «Pour rendre cette action plus légère et positive, il vaut peutêtre mieux penser "élever" comme "instaurer", et non comme "éduquer" ".

Les mots de Jean-Christophe sont justes, «justes» dans le sens où ils touchent avec beaucoup de tact, l'intelligence de l'expérience qu'il relaie. Car c'est bien comme lectrice de Souriau que je comprendrais également ce que font ces parents: "élever» une enfant, instaurer son existence et la promouvoir, et continuer à la faire grandir. Ils la font persévérer dans l'être. Cette existence nouvelle, qui prolonge celle que Juliette avait commencée, est exigeante, elle demande des soins, de l'inventivité et de l'attention, grâce auxquels elle peut s'épanouir. Car avec cette boîte aux lettres, Juliette continue à vivre pour d'autres, et même à exister pour d'autres qu'elle n'a pas connus dans sa première vie et qui participent activement à soutenir

3. Documentaire autobiographique d'Éric Caravaca, réalisé en 2017. Entre la France et le Maroc, le comédien-cinéaste cherche à retrouver les traces de sa sœur aînée, Christine, décédée à l'âge de trois ans et dont plus rien ne subsiste, si ce n'est le «caveau 35» dans le cimetière de Casablanca où elle repose. 
l'existence dont elle bénéficie à présent. Elle reçoit ce supplément de biographie qui lui permet de nouer de nouvelles relations ou d'en prolonger d'anciennes, qui lui donne la possibilité, en somme, d'être présente (au sens également d'être conjuguée au présent) et de continuer à agir dans d'autres vies.

Mais ce que ces gestes et les récits qui les prolongent font particulièrement sentir, c'est la nécessité de maintenir, vivante et active, l'indétermination. Ils mettent en œuvre, pour ce faire, tout un attirail technologique pour la préserver. Ils font, en d'autres termes, ce que la clinicienne Magali Molinié inspirée par Deleuze nomme, pour qualifier sa propre démarche auprès des endeuillés: "suivre par le milieu» (2006: 137). Suivre par le milieu, précise-t-elle, c'est aborder la question de telle sorte à ne perdre de vue ni les vivants ni les morts, c'est apprendre à les suivre ou à les rencontrer par ce qui les lie, par ce qui «les tient ensemble». C'est ce que font donc les récits : protéger la polyphonie des versions. Penser par le milieu, ce que font les personnes qui fabriquent ces récits, c'est aussi ne pas séparer ce sur quoi on s'interroge - une expérience, un être, une présence -, du milieu qu'il requiert pour exister. Ce type de récits, en ce sens, constitue, chez nous, une des niches écologiques privilégiées, non la seule, mais une des niches possibles. L'attirail technologique qui réunit les conditions du maintien de l'existence est divers. Il y a des actes, qui nourrissent des récits et qui s'y alimentent: choisir des chaussures avec soin; préparer pour une épouse aimée, le jour de son anniversaire, le repas qu'elle préférait ; écrire des lettres adressées à l'absent; dédier une œuvre, continuer ce qu'il ou elle faisait et qui n'a pu être achevé; accueillir des présences; parler, rêver, chercher les signes et construire, à partir de ces expériences, et avec beaucoup de soin, leurs récits. Et pour ceux-ci, comme je viens de l'évoquer, il s'agira, à chaque fois, d'un petit bijou d'invention linguistique, de telle sorte à maintenir tous les possibles ouverts, avec, par exemple, l'ingéniosité de la construction formelle en termes de "comme si", "comme s'il avait voulu me faire un signe», particulièrement à même de soutenir la perplexité qui nourrit l'expérience.

Ainsi, l'expression «comme s'il avait voulu me faire un signe», qui revient si souvent sous une forme ou sous une autre, laisse ouverte la question de l'intention du défunt, et intacte l'incertitude quant à savoir si le signe est le produit de l'interprétation de celui qui le reçoit, ou s'il émane "vraiment» du défunt. Il y a bien entendu également l'usage de métaphores, voire l'invention de nouveaux sens aux mots. Il y a, plus ouvertement, la technique de l'oscillation entre deux versions contradictoires: «Était-ce dans ma tête, était-ce autre chose?». Le récit, de ce fait, par l'indétermination qu'activement il préserve, est maintenu vivant. Car justement, le danger 
auquel il échappe, c'est le mot de la fin. C'est ce que j'ai appris à appeler, le «tact ontologique». C'est ce tact que je retrouve dans la réponse que donne le jeune Rachid à la question que lui pose l'une de mes étudiantes en psychologie, à savoir si parfois il sentait la présence de son jeune frère décédé dans un accident de moto:

"Oui plusieurs fois, par des ombres, un bruit, le rêve. Par les objets qui lui appartiennent aussi. Dès que je ressens sa présence, j'accepte d'abord cette présence et j'essaye de laisser les portes et les fenêtres ouvertes voire, même, j'ouvre les tentures pour laisser la lumière pénétrer. Je n’ai pas peur ou des trucs dans le genre, je reste plutôt passif et j'essaye de faire avec. Lorsque je ressens sa présence, j’ouvre même la fenêtre pour quil puisse sortir sìl le désire» (Doutrelpont 2012).

On sent dans cette narration, une gradation savamment orchestrée de la distribution de ce qu'on appelle l'«agentivité» ou l'«agence», et que je traduis, à la suite de Bruno Latour, comme la puissance d'agir: des ombres, du bruit, un rêve, des objets qui appartiennent au défunt d'abord, et qui ne font qu'évoquer la présence; puis la lumière, et les fenêtres ouvertes, qui préparent non plus l'évocation mais une invocation; enfin, la fenêtre ouverte pour le laisser sortir s'il le désire témoigne, avec un tact remarquable, d'un rituel de convocation étonnamment sophistiqué. Rachid crée la situation qui permettra à ce désir d'exister, et ce désir gagne d'autant plus d'autonomie qu'il pourrait s'avérer être de ne pas donner suite à la convocation, ou plus justement, de la décliner.

Mais restait une modalité que je n'avais pas prise en compte, pendant mon enquête, ou qui m’avait étrangement échappé: celle de prolonger le récit par le moyen le plus simple: l'ouvrir à d'autres, mais cette fois sur le mode de la fiction. Et offrir, par cette dernière, le supplément biographique que le mort semble réclamer. Une dame m'a envoyé un courriel pendant l'été 2016, et voici ce qu'elle y racontait:

«Ma mère, Lore, est décédée il y a plus d'un an, le 7 mai 2015. De son vivant, elle avait exprimé la volonté d'être incinérée, au contraire de mon père inhumé 17 ans auparavant. Grande voyageuse, ayant travaillé dans l'aviation de 1958 à 1970, amoureuse des déserts, ma maman avait par le passé exprimé à plusieurs reprises le souhait que ses cendres soient dispersées dans le désert. À travers nos derniers échanges, j’avais compris que le désert qui pourrait idéalement l'accueillir serait celui du Namib. Ce serait donc celui-là, sans qu'elle me l'ait d'ailleurs (jolie formule :-) expressément demandé. Le désert du Namib et son sable orangé l'avaient marquée, le dernier qu'elle avait découvert un mois avant la naissance de son unique petit-enfant, le plus vieux désert du monde, "le plus spécial” selon ses propres termes. Sans parler des connections diverses avec ce pays, ancienne colonie allemande, qui ne pouvaient que séduire sa personnalité ambivalente et déracinée, ma maman étant née en Allemagne puis s'étant expatriée en France. Pour moi, l'an dernier, ce fut une évidence. Sa destination finale devait être celle-là, je (me) devais répondre à cette demande implicite, ce serait dès lors son dernier voyage. Et à 
travers celui-ci, elle me ferait découvrir d'autres horizons, une autre perspective... pour assurer un relais, question de transmission. J'ai donc entamé au cours de l'été 2015 les démarches pour visiter avec nos proches (mon fils et mon compagnon) la Namibie et l'y emporter. Il serait plus juste de dire que je me suis engagée dans un véritable parcours d'obstacles. J'ai entrepris et accompli toutes les formalités requises, voire même au-delà, obtenu toutes les autorisations nécessaires pour que le transfert de l'urne funéraire se fasse sans encombre. Puis j'ai attendu la date du départ. Cette attente fut longue, j'ai affectivement énormément investi ce voyage. Une fois en Namibie, enfin (the end ? eh bien non), nous nous sommes hélas fait dérober, dès le deuxième jour de notre séjour, une partie de nos bagages dans "un vol à la roulotte". Nos valises étaient dans le coffre de notre véhicule de location, garé en ville et fermé. Parmi les sacs dérobés se trouvait... l'urne contenant les cendres de ma maman! Le 7 juin 2016, soit 13 mois jour pour jour après qu'elle ait expiré son dernier souffle. Elle s'est ainsi littéralement "envolée" hélas pas dans le sens où je l'entendais. Hélas pour qui ? Pour elle? Pour moi? Passés le choc et l'effondrement émotionnel qu'a occasionnés cette mésaventure surréaliste, je cherche désormais quel sens donner à cette histoire. Ce récit peut sembler rocambolesque, digne d'un scénario. Il ne l'est absolument pas, il touche à quelque chose d'essentiel et de profond. Je le perçois même si je ne parviens pas encore à prendre le recul véritable par rapport à l'événement. J'ai bien entendu des pistes de réflexion, quelques convictions et intuitions, mais je me bats parfois avec des sursauts de détresse intense lorsque ce vol me revient à l'esprit. Sur place, j'ai porté plainte, la police namibienne n'a rien retrouvé à ce jour et à ma connaissance, le voyagiste a fait des appels à la radio et via les médias sociaux. Je dois désormais accomplir un signalement auprès du Quai d'Orsay au cas où l'urne serait retrouvée dans l'avenir en raison de la législation relative à la protection des corps! De fait, je me suis sentie dépossédée, ma démarche est restée inachevée, je n'ai pas conduit ce projet intime et personnel à son terme... Ma maman a certes atterri sur le sol namibien mais où sont ses cendres aujourd'hui ? [...] Ayant quitté avec regrets (un euphémisme) la Namibie, je m’interroge. Aurais-je dénaturé la volonté de ma maman ou, au contraire, s'est-elle échappée pour gagner définitivement une forme de liberté? Elle s'était définie en fin de vie comme "un oiseau migrateur"... M'invite-t-elle de mon côté à lâcher prise?».

J'avoue que je trouvais l'idée d'une fugue tellement jolie que j'ai répondu par retour de courriel en ce sens. Ce que je qualifiais de "magnifique échappée posthume» m'apparaissait en même temps, dans l'idée de ma correspondante d'une invitation à lâcher prise, comme une leçon de liberté. Les dernières volontés, on l'oublie souvent, demandent une suite. C'est un des sens possibles à ce que nous appelons hériter - on devrait d'ailleurs plutôt parler d' "avant-dernières volontés ", charge aux survivants de veiller aux dernières. Aussi ma correspondante me répondit-elle peu de temps après qu'elle s'était inscrite à un atelier d'écriture, prête cette fois à donner cette suite sur le mode de la fiction, et offrir à sa mère, ce que Souriau considérerait peut-être comme une promotion dans l'existence. Le vol ne serait donc pas le mot de la fin. 
L'histoire de Lore, morte voyageuse, pourrait trouver écho dans la très belle recherche qu'a menée Arnaud Esquerre (2011) sur la circulation des cendres. En fait, grâce au vol, les cendres de Lore ont bénéficié de bien plus de liberté que ne l'autorise la loi française depuis 2008 - preuves de ces restrictions de liberté à la fois les difficultés administratives du voyage et la nécessité d'avertir le Quai d'Orsay. Moins de dix ans auparavant, ma correspondante aurait échappé à toutes ces tracasseries administratives. En effet, entre 1976 et 2008, explique Esquerre, avec la généralisation de la crémation et la relative absence d'encadrement législatif, des milliers de morts ont circulé librement sur le territoire parmi la population, ce qui conduit par ailleurs Grégory Delaplace (2015) à souligner que la place des morts devrait se poser moins en termes de lieux qu'en termes de trajectoires. Mais plus étonnant encore, apparaît, à la lecture de l'enquête d'Arnaud Esquerre, l'étrange complexe de pensées qui a régi le changement de loi. Je voudrais m'attacher à deux des arguments qui ont été avancés. Pour introduire le premier, je voudrais revenir quelques instants à la comparaison qui ouvrait mon exposé, celle entre tous ces êtres problématiques appelés à être des gens comme les autres. Faute de temps, je laisserai les chiens de côté pour évoquer à nouveau le Néanderthal que Svante Pääbo voudrait protéger d'une tentative de clonage. À la fin de son argumentaire, Pääbo en appelle à son grand-père, comme à Néanderthal, et termine par cette phrase: "Qu'on les laisse reposer en paix». Et c'est bien une réclamation récurrente, que l'on a pu retrouver, par exemple, comme le souligne l'historien Jay Winter, à la fin de la Première Guerre mondiale, lorsque les politiques, dans leurs discours, invoquaient les morts pour restaurer l'ordre social, les prenant en otage de conflits dont ils étaient résolument étrangers. Cela suscitera la colère de nombreux soldats, dont le poète Marc de Larreguy demandant, au nom des camarades qu'il rejoindra d'ailleurs peu après dans la mort, «qu'on les laisse enfin pourrir tranquille» (cité in Winter 2008 [1995] : 229). Ne doit-on pas laisser les morts tranquilles, pour reprendre, en le détournant quelque peu, le titre d'un article de Pascal Boyer (2016) ? Ou encore, dans la version primesautière d'Emmanuel Berl, «Si on croit que les morts survivent, comment croire qu'ils ne font rien, qu'ils n'ont rien à faire, qu'on ne les dérange jamais?» (1995 [1956] : 80). Retournons à l'enquête d'Arnaud Esquerre: l'argument de la tranquillité des morts, étonnant en ce début du $\mathrm{XXI}^{\mathrm{e}}$ siècle, semble pouvoir convaincre de la nécessité d'une réglementation sur leur circulation. La fête est finie. Ou pour reprendre une des formulations utilisées par un rapporteur de l'Assemblée nationale, «le principe de la paix des morts impose [...] que la sépulture choisie soit stable» (cité in Esquerre 2011: 79). L'enjeu juridique, en fait, est d'identifier les lieux où reposent les morts, de les immobiliser et de les rendre accessibles à tous. 
Intervient ici un second argument, assez étrangement associé au premier, puisqu'il puise dans le fond de la doxa psychologique et s'apparente à ce qu'on pourrait appeler le «nouveau régime des obligations de deuil». En effet, l'éventualité d'une appropriation des cendres, ou de leur disparition, menace, selon le législateur (et selon nombre de ceux qui ont pris position dans cette affaire, dont les représentants de l'Église), la possibilité pour les endeuillés de faire le travail de deuil. Et l'on pourra donc conclure, tant avec Arnaud Esquerre qu'avec la sociologue Dominique Memmi (2014), que l'enjeu déclaré s'avérait bien la protection du psychisme des vivants.

Je ne reviendrai pas sur ce que le psychanalyste Jean Allouch (1997), la psychologue Magali Molinié et quelques autres ont dénoncé comme l'absurdité de ce «travail de deuil » et qui n'est pas sans évoquer une énième tentative de domestication des psychés. L'incongruité apparente qui consiste à accorder aux morts une volonté, celle d'être en paix, et, en même temps, à invoquer un système théorique qui affirme que lorsque les morts sont morts, ils sont morts et donc ne doivent plus rien désirer, ne l'est peut-être pas tant que cela, incongrue, si on songe que ces deux injonctions, finalement, rappellent une même attitude: celle de vouloir clairement maintenir la séparation des vivants et des morts. Si les laisser en paix signifie les laisser être morts, et si le travail du deuil, comme prescription, implique bien l'injonction de s'en séparer, les arguments relèvent finalement d'une même règle de socialité, pour les morts comme pour les vivants. Cela me semble d'autant plus plausible, pour l'enrôlement de la théorie du deuil comme témoignant de la volonté de l'État de reprendre à son compte l'injonction de séparation pour protéger le psychisme de ses citoyens, que l'un des arguments en faveur de la mise à la disposition de tous des cendres du défunt a reposé sur une série d'anecdotes malheureuses, par exemple le fait que la détention, à domicile, de l'urne des cendres d'une première épouse avait entraîné la demande de divorce de la seconde, ou bien encore, l'obligation, pour un enfant, de saluer tous les matins son petit frère décédé.

On pourrait s'attendre à ce que pour les cercles spirites, dont les pratiques s'avèrent aller totalement à rebours de la théorie du deuil, la question de la tranquillité des morts ne soit pas posée puisque, loin de favoriser la séparation, les pratiques ne cessent de susciter les contacts. Or, une analyse des séances me semble au contraire montrer une préoccupation certaine des médiums à l'égard du fait que les vivants peuvent, par moment, entraver le destin de leurs proches décédés. Précisons que ces séances, du moins dans le cercle où je me suis régulièrement rendue pour mon enquête, sont collectives, organisées deux fois par semaine dans une salle accueillant généralement une trentaine de personnes. À l'entrée, les participants peuvent, s'ils le souhaitent, déposer une photographie du défunt avec lequel ils 
voudraient entrer en contact et qui sera remise aux deux médiums officiant. Ce qui n'empêche pas les visites intempestives de morts non convoqués par une photographie. C'est même assez souvent le cas, que ce soit pour des habitués, que finissent par bien connaître les médiums, ou pour des personnes de passage. Je voudrais évoquer deux situations, mais bien d'autres pourraient recevoir cette même lecture, où il m'est apparu clairement que le travail de Michèle et de Philippe a consisté à dénouer des liens trop serrés ou mal tissés, et ce, au nom du bien-être des morts. Michèle s'adresse à un homme d'une quarantaine d'années, sur un ton de reproche. Sa femme est là, dit-elle. Elle n'est pas bien. "Il semble que vous vous complaisez dans le malheur». Il s'exclame: "Me complaire dans le malheur! Mais elle en a de bonnes celle-là!». Tout le monde rit. Michèle continue: «Mais vous rendez votre femme malheureuse, votre femme se sent coupable. On ne peut pas culpabiliser les morts. Ce n'est pas de leur faute d'être parti, c'était leur heure, on n'y peut rien. Et votre femme se sent coupable parce que vous la rendez coupable». Se manifeste dans cet échange une inflexion subtile de la théorie du deuil. Et cette inflexion en inverse radicalement le rapport. Il ne s'agit pas de se détacher du disparu pour réinvestir d'autres objets, se «libérer de sa férule», comme disent certains. Non, c'est la morte qui, ici, demande à être libérée. S'il apparaît bien, avec cette inversion, que le destin du mort est au centre des préoccupations des médiums, on ne manquera toutefois pas de remarquer que ce qui est également en jeu ici, et que la médium déjoue, c'est l'impossibilité pour un vivant de prendre la très difficile décision, la décision parfois impossible, de renvoyer le mort à une forme de non-existence en se débarrassant d'un chagrin qui témoigne du fait qu'il a bien été, du chagrin qui le maintient dans l'être. C'est là le coup de génie du dispositif, si l'ordre vient de la morte elle-même, si l'obligation de renouer avec la vie émane d'elle comme une nécessité, il peut être entendu. C'est ce qui me conduit à dire, ou à redire ici, que le dispositif spirite est un dispositif thérapeutique. L'autre situation concernait un jeune homme venu avec la photographie de son ami. Celui-ci s'était donné la mort quelques mois auparavant. Avec beaucoup de tact, Philippe lui a d'abord dit qu'il ne pouvait être en contact avec lui, car il était trop mal, et que ce sont d'autres êtres qui lui répondaient. Il a continué:

«On me donne quelqu'un qui était très optimiste, une vision un peu naïve, mais progressivement, il s'est passé quelque chose, il est passé de désillusions en désillusions, et il n'y avait plus rien d'intéressant ou de beau. Déjà, de son vivant, il a dû s'isoler. Vous vous souvenez de lui quand il était encore bien? [le jeune homme répond que oui]. Si vous pensez à lui quand il était encore bien, cela pourrait l'aider à se dégager. Il est comme dans un cocon enfermé, ce sont d'autres entités qui me renseignent. C'était quelqu'un de très gentil, d'hypersensible. Il s'attendait à ce que le monde soit comme 
lui. Ce sont vraiment des désillusions qui sont venues progressivement. Ce n'est même pas de la culpabilité, il n'en est pas encore là. Quand on pense à lui, on pense à la fin et cela le fige encore plus. Cela vous demande une démarche active de le voir heureux et souriant. Il est nécessaire et utile de lui envoyer des pensées positives et de le visualiser heureux et joyeux. Si on vous a poussés à amener sa photo, c'est qu'il y a quelque chose à faire pour lui. Il faudra revenir avec sa photo, dans quelque temps».

La description du clairvoyant est juste (je connaissais aussi bien le jeune homme que son ami), ou plutôt - laissons là ce que nous savons et qui nous situerait dans un régime de vérité qui n'est peut-être pas le plus adéquat dans ce cadre - elle touche juste. Car la question n'est pas de savoir s'il dit vrai ou non, la question est de comprendre ce que sa proposition touche, et ses effets. Elle fait sens, elle devient vraie, elle reconstruit l'histoire sur un mode tel qu'elle donne à présent une prise. Ce qui n'a pu être fait peut encore l'être. Cette proposition met le jeune endeuillé dans une autre posture à l'égard du disparu, surtout, elle transforme le régime de l'action et des affects, dans le même geste: elle le fait passer d'un régime passif à un régime d'activité, et de passions tristes en passions joyeuses. Le vivant, ainsi activé et touché, repart avec une responsabilité: reconstruire le passé, activement, pour ouvrir d'autres possibles dans le futur. Agir et transformer des manières d'être, non pas rétrospectivement, mais rétroactivement.

La prise que j'ai choisie ne serait certainement pas celle par laquelle les clairvoyants rendraient compte de leur pratique, ce qui pose évidemment la question de la fidélité et de l'interprétation. Paul Sorrentino a rencontré un problème relativement similaire avec les chercheurs vietnamiens travaillant au Centre de recherche sur les potentiels humains, à Hanoï. Ceux-ci s'efforcent de montrer la véracité des compétences que met en œuvre la possession. Malgré leur intérêt partagé pour cette dernière, Sorrentino remarque que ces chercheurs sont beaucoup plus réticents à accepter son idée selon laquelle leurs activités elles-mêmes auraient contribué au développement des pratiques de possession par les morts (2018: 155, note 19). Qu'ils aient le sentiment que cette lecture constructiviste puisse aller à l'encontre du régime de la preuve qui guide leur travail est tout à fait compréhensible, puisque ce régime de la preuve exige l'authenticité des mécanismes de possession, une authenticité qui se verrait irrémédiablement entachée de suspicion si on en venait à croire que leur recherche pourrait être la cause du phénomène qu'ils étudient et qu'ils promeuvent du fait de l'étudier. Mais Sorrentino ne peut adhérer à ce régime de la preuve, car cela l'obligerait à accepter une définition scientiste de la vérité: n'existe vraiment que ce qui existe "par soi-même». La prise constructiviste qu'il choisit, en acceptant que le travail de l'enquête est partie prenante du phénomène que les enquêteurs observent, lui permet de ne pas statuer sur l'exacte teneur en réalité de ce 
qu'il étudie, et d'échapper au choix comminatoire entre «c'est construit» et "c'est réel». Non, comme Bruno Latour nous l'a appris, une fois qu'on se débarrasse de la définition scientiste des sciences, le phénomène est d'autant plus réel qu'il est bien construit. À l'enquêteur d'étudier alors comment c'est construit, et surtout, comment ça tient. Mais le problème de la vérité ou de l'authenticité n'est pas un problème abstrait pour ces chercheurs vietnamiens: ce centre de recherches a dû affronter quantité de difficultés pour obtenir de l'État la possibilité de mener ses travaux, pour leur offrir un cadre légal et ne pas tomber sous le coup des lois contre les superstitions (Sorrentino 2018: 157). Comment à la fois ne pas les mettre en danger mais également répondre aux exigences de sa propre pratique? C'est là que le chercheur se trouve sans doute dans la position du diplomate que met en scène Isabelle Stengers $(2003,2006)$ : il s'agit d'apprendre à penser avec les autres, mais tout en sachant qu'il faut revenir vers les siens. On est loin, ici, du mot de la fin.

Rassurez-vous, en ce qui concerne cette conférence, nous y sommes presque. Mais je m'en voudrais, revenant à Michèle et Philippe, s'il s'agissait de penser la difficulté que peut poser le fait de comprendre le travail des médiums comme un travail thérapeutique, de ne pas me faire aider par la ressource extraordinaire qu'est, pour nombre d'entre nous, le travail de Jeanne Favret-Saada. L'anthropologue Jérémy Damian (2014: 528) rapporte que lorsqu'elle revient, quelques décennies plus tard, sur le dispositif de désorcèlement qu'elle avait étudié dans le Bocage, en choisissant de le présenter comme un "dispositif thérapeutique» de cure et de présenter les désorceleurs comme des «thérapeutes» ${ }^{4}$, elle suppose que:

«[...] l'accueil d'une telle proposition ne se fera pas dans le sens qui aurait, en apparence, paru le plus évident: des désorceleurs comblés d'être enfin reconnus comme de véritables et efficaces thérapeutes. Elle précise en effet que les "vrais" thérapeutes - ceux qui ont l'habitude de se présenter comme tels - seraient finalement plus enclins à accepter les désorceleurs en leur sein que les désorceleurs ne le seraient à se reconnaître appartenir au cercle des thérapeutes" (Ibid.: 529).

Jérémy Damian a repris le problème à propos de son propre terrain avec des praticiens de «danse improvisation contact» et montre que ce qui pourrait être vécu comme une trahison pour les désorceleurs s'avère, et ce sont ici mes termes, un compromis équitable. Car de fait, Jeanne Favret-Saada choisit délibérément de ne pas opérer le geste critique, celui qui consisterait à faire bifurquer le réel entre, d'une part, des apparences, des illusions, de la subjectivité ou des projections et, de l'autre, une réalité «vraie » à laquelle le

4. Cf. en particulier les chapitres II, «La thérapie sans le savoir», et III, «L'invention d'une thérapie» (Favret-Saada 2009: 24-77). 
chercheur critique aurait seul accès. Pas plus qu'elle ne renvoie, si ce n'est exceptionnellement, à une interprétation symbolique de la sorcellerie - ce qui serait l'alternative «acceptable» pour la vulgate anthropologique -, elle note d'ailleurs que la référence au symbolique n'est jamais aussi aisément convoquée que lorsque l'observateur se trouve confronté à des énoncés qu'il considère comme faux (2009: 149). Elle offre ainsi à son terrain une "prise» pragmatique en termes de ce que «fait» le désensorcèlement et de ce qu'il permet aux gens de faire. S'il lui apparait intéressant de s'autoriser à ne pas avoir la même grille de lecture que les désorceleurs, écrit Jérémy Damian, "c'est que cela lui permet de se dégager de l'alternative qui tient en tenaille l'efficace de ces pratiques entre "y croire" ou "ne pas y croire". Sans n'émettre aucun jugement, sans discréditer aucune expérience, elle peuple le monde d'une pratique intéressante supplémentaire» (2014: 529). Et Damian de rappeler ce que Viveiros de Castro assignait comme tâche à l'anthropologie: "S'il y a quelque chose qui revient de droit à l'anthropologie, ce n'est pas la tâche d'expliquer le monde d'autrui, mais bien celle de multiplier notre monde" (2009: 169).

La question de la divergence se pose autrement encore pour moi, puisque finalement ma prise pratico-théorique est beaucoup plus proche de celle des médiums que ne l'est la pratique du désensorcèlement de celles des thérapies conventionnelles. Philippe et Michèle savent qu'en aidant les morts, ils aident les vivants. Et ils savent qu'il faut aider les vivants à prendre soin des morts. La lecture que je leur propose est en réalité très similaire en apparence à la leur, sauf que la mienne inverse radicalement la perspective. Elle déplace le focus des morts sur les vivants, non pour nier la possible implication des morts, mais parce que je suis obligée de concéder que là, mon savoir s'arrête. Je ne peux pas savoir s'ils aident les morts, ni si ceux-ci sont concernés par ces histoires. Mais je peux voir, et être sensible, au fait que ce que les médiums proposent non seulement aide ceux qui restent, ce qui n'est pas rien, mais qu'en outre, c'est passionnant. Et que le monde qu'ils contribuent à faire exister et dans lequel ils œuvrent est bien peuplé. Eduardo Viveiros de Castro disait encore de l'anthropologie qu'elle étudie les variations d'importance. Michèle et Philippe font importer plus d'êtres pour qui des vivants importent. Ce n'est pas tout. Mais c'est déjà très important.

Université de Liège Département de philosophie, Liège (Belgique) V.Despret@uliege.be 
MOTS CLÉS/KEYWORDS: médium/medium - spiritisme/spiritism- mort/death - deuil/grief.

Allouch, Jean

1997 Érotique du deuil au temps de la mort sèche. Paris, EPEL.

\section{Bergé, Christine}

1990 La Voix des esprits. Ethnologie du spiritisme. Paris, Métailié («Traversées»).

Berl, Emmanuel

1995 [1956] Présence des morts.

Paris, Gallimard («L'Imaginaire»).

Bloch, Maurice

1993 «La mort et la conception

de la personne», Terrain 20:7-20.

\section{Boyer, Pascal}

2016 «Pourquoi ne pas laisser les morts tranquilles? Personnes contre-intuitives", in Aude Gros de Beler, ed., Persona.

Etrangement humain. Paris, Musée du quai Branly/Arles, Actes Sud: 55-57.

\section{Charuty, Giordana}

2002 " "Ça passe très vite et je n'y peux rien": séances médiumniques dans une salle de voyance parisienne", Vacarme 20 (3) : 73-74. En ligne: https://www.cairn.info/ revue-vacarme-2002-3-page-73.htm

Damian, Jérémy

2014 Intériorités/sensations/consciences. Sociologie des expérimentations somatiques $d u$ Contact-Improvisation et du BodyMind Centering. Grenoble, Université de Grenoble-ED SHPT, thèse de doctorat.

\section{Delaplace, Grégory}

2015 «Incertitudes morales, régimes de visibilités et vitesse de circulation des morts ", in Grégory Delaplace \& Fréderique Valentin, eds, Le Funéraire. Mémoires, protocoles, monuments. Paris,

De Boccard ("Colloque de la Maison RenéGinouvès») : 11-23.

\section{Esquerre, Arnaud}

2011 «Les morts mobiles: étude sur la circulation des cendres en France», Raisons politiques 41 (1) : 69-85.

Favret-Saada, Jeanne

2009 Désorceler. Paris, L’Olivier ( Penser/Rêver»).

\section{Féry, Bernard}

2018 La Veuve du procureur. Paris, Persée.

James, William

1913 [1909] L'Idée de vérité. Trad. de L. Veil et M. David. Paris, Félix Alcan («Bibliothèque de philosophie contemporaine»).

1948 [1911] Some Problems of Philosophy. A Beginning of an Introduction to Philosophy. New York, Longmans, Green, \& Co.

\section{Kwon, Heonik}

2008 Ghosts of War in Vietnam. Cambridge, Cambridge University Press.

Memmi, Dominique

2014 La Revanche de la chair. Essai sur les nouveaux supports de l'identité. Paris, Le Seuil («La Couleur des idées»).

\section{Michelet, Aude \& Charles Stépanoff}

2016 «Comment l'anthropomorphisme nous a rendus humains: l'anthropomorphisation des animaux et des nourissons et ses impacts dans l'évolution ", in Aude Gros de Beler, ed., Persona. Étrangement humain.

Paris, Musée du quai Branly/Arles, Actes Sud: 45-50.

\section{Molinié, Magali}

2006 Soigner les morts pour guérir les vivants. Paris, Les Empêcheurs de penser en rond. 


\section{Pons, Christophe}

2002 Le Spectre et le voyant. Les échanges entre morts et vivants en Islande. Paris, Presses de l'Université de Paris-Sorbonne ("Voix germaniques»).

\section{Sorrentino, Paul}

2018 À l'épreuve de la possession. Chronique d'une innovation rituelle dans le Vietnam contemporain. Nanterre, Société d'ethnologie («Recherches sur la Haute Asie»).

\section{Souriau, Étienne}

1939 L'Instauration philosophique. Paris, Félix Alcan («Bibliothèque de philosophie contemporaine»).

\section{Stengers, Isabelle}

2003 Cosmopolitiques, 2. Mécanique quantique, Au nom de la flèche du temps, La vie et l'artifice, Pour en finir avec la tolérance. Paris, La Découverte ( $«$ La Découvertepoche. Sciences humaines et sociales»).

2006 La Vierge et le neutrino.

Les scientifiques dans la tourmente. Paris, Les Empêcheurs de penser en rond.
2007 «Préface», in Tobie Nathan, Nous ne sommes pas seuls au monde. Les enjeux de l'ethnopsychiatrie. Paris, Le Seuil («Points. Essais» 575).

2014 "Penser à partir du ravage écologique», in Émilie Hache, ed., De l'univers clos au monde infini. Bellevaux, Dehors: 147-190.

\section{Viveiros de Castro, Eduardo}

2008 «Claude Lévi-

Strauss, Euvres», Gradhiva 8: 130-135.

En ligne: http://journals.openedition.org/ gradhiva/1215

2009 Métaphysiques cannibales. Lignes d'anthropologie post-structurale. Trad. du portugais (Brésil) par Oiara Bonilla. Paris, Presses universitaires de France («MétaphysiqueS»).

\section{Winter, Jay}

2008 [1995] Entre deuil et mémoire.

La Grande guerre dans l'histoire culturelle de l'Europe. Trad. de l'anglais par Christophe Jacquet. Paris, Armand Colin. 
